\title{
Save the males!
}

\author{
David C. Page
}

Howard Hughes Medical Institute, Whitehead Institute, and Department of Biology, Massachusetts Institute of Technology,

9 Cambridge Center, Cambridge, Massachusetts 02142, USA

Why has the $\mathrm{X}$ chromosome always received such a disproportionate share of attention from human geneticists? Probably not because it is a middling-sized chromosome with an apparently below-average gene density ${ }^{1}$ and a modest level of sequence polymorphism. Perhaps because it has the good sense to pair and recombine with the noble Y chromosome during male meiosis? Probably not. Do the mysteries of dosage compensation and $\mathrm{X}$ inactivation seduce? These are interesting subjects, but are not the focus of most X-linked publications. The human X chromosome has been studied intensely because it is linked to a large number of heritable traits and disorders that almost exclusively affect males. Nearly two thousand years ago, the writers of the Talmud understood the concept of hereditary, male-limited disease transmitted through unaffected females, exempting boys from circumcision when two or more male relatives had died of excessive bleeding ${ }^{2}$.

The foremost X-chromosome paradigm is the $\mathrm{X}$-linked recessive mode of inheritance, exemplified by more than 300 traits $^{3}$. Males are monosomic for the $\mathrm{X}$ chromosome, whose alleles are thus permitted to fly without copilots; phenotypic mishap is frequent. In aggregate, $\mathrm{X}$-linked recessive disorders (for example, colour blindness and haemophilia) may affect 8 to $10 \%$ of the male population. From the perspective of sexual politics and scientific priorities, how big a stipend would the sex-linked recessive disorders draw, were the Y-borne $S R Y$ gene a dominant female determiner rather than a dominant male one? In such a case, sex-linked recessive disorders would afflict females, and not males.

The tables have now been turned in the case of a family in which an X-chromosomal mutation causes disease only in females. As Stephen Ryan and colleagues demonstrate in this issue ${ }^{4}$, epilepsy and mental retardation (EFMR) in this family are inherited as $\mathrm{X}$-linked dominant traits with male sparing. In other words, heterozygous $(-/+)$ females are affected, but hemizygous $(-/ \mathrm{Y})$ males are not. (No homozygous (-/-) female has been or is likely to be described.) In the case of traditional X-linked recessive disorders, carrier females transmit the trait to half of their sons. In the present family the carriers are phenotypically normal males who transmit the disorder to all, or nearly all, of their daughters; penetrance is high but not complete. The pattern of EFMR inheritance should not be confused with that of the previously reported, so-called 'X-linked dominant' disorders like incontinentia pigmenti and focal dermal hypoplasia, where $-/ Y$ male fetuses die in utero and $-1+$ females, whose development is less severely perturbed, exhibit a clinically recognized, postnatal phenotype. In the case of the family studied by Ryan and colleagues, $-/ Y$ males appear to be phenotypically normal.

How can this be? By what mechanism are the males spared? In the case of autosomal genes, sex-limited or sex-biased phenotypic expression can usually be traced to the far-flung regulatory consequences of sex steroids (androgens and estrogens), whose levels differ dramatically between males and females, both before and after birth. Thus, sex steroids account, albeit indirectly, for the disproportionate occurrence of baldness among males and of breast cancer among females, in genetically predisposed populations. Indeed, hairy ear rims in some males, often attributed to a Y-chromosomal gene, more likely reflect the influence of steroids on the expression of autosomal loci. Sex steroids could also account, directly or indirectly, for femalelimited expression of X-linked epilepsy and mental retardation in the present family, as Ryan and colleagues acknowledge.

The authors also present an alternative hypothesis that is particularly intriguing. They speculate that in $-1+$ females, the disease is caused by the absence of wild-type function in half of the cells (a result of the gene in question being subject to $\mathrm{X}$ inactivation); and that in -/Y males, the X-chromosomal defect is complemented by a functional, homologous gene on the $Y$ chromosome. In considering this hypothesis, it is important to recognize two stumbling blocks, the first of which is that EFMR maps to Xq21-q24, far from the telomeric, pseudoautosomal regions where $X-Y$ recombination is a normal and frequent event during male meiosis, and where the majority of $\mathrm{X}-\mathrm{Y}$ common genes are likely to be found. However, the portion of $\mathrm{Xq}_{\mathrm{q}}$ implicated by the linkage studies of Ryan and colleagues does contain a block of approximately $4 \mathrm{Mb}$ with Y-homology; probably the largest block of Y-homology anywhere on the $\mathrm{X}$ chromosome $\mathrm{e}^{5-7}$. No transcription units have yet been identified within this $X-Y$ common region, and no trait has been unambiguously mapped within it. Perhaps EFMR will be the first.

In addition, in all but one of the many $X-Y$ gene pairs examined to date, the $X$ linked genes appear to escape $\mathrm{X}$ inactivation $^{8,9}$; this is directly at odds with the authors' assumption - that EFMR undergoes $\mathrm{X}$ inactivation. However, this particular X-Y common block is unusual in that it resulted from a duplicative, $\mathrm{X}$-to- $\mathrm{Y}$ transposition that occurred only three million years ago, during hominid evolution (ref 5; Schwartz et al., unpublished results). Prior to this recent, massive acquisition by the $Y$ chromosome, these X-chromosomal sequences were perfectly ordinary; they had no $Y$ homologues and probably underwent $\mathrm{X}$ inactivation. Given the recent nature of the transposition, there has been little opportunity for the X-chromosomal genes to evolve - for example, to escape $X$ inactivation in response to the existence of $Y$ homologues. Thus, genes in this largest block of Y homology probably remain subject to $X$ inactivation, in keeping with Ryan and colleagues' $\mathrm{X}-\mathrm{Y}$ hypothesis.

If this X-Y hypothesis proves correct (it is highly speculative conjecture at present) then the X-to-Y transposition that occurred during hominid evolution was an act of prophylactic gene therapy, protecting males in the present family, three million years later, from mental retardation and epilepsy. Perhaps in this instance, an evolutionary event accomplished what a century of human genetics has yet to do for most sexlinked disorders - to save the males.

1. Schuler, G.D. et al. Science 274, 540-546 (1996)

2. Stern, C. Principles of Human Genetics (W.H. Freeman, San Francisco, 1973).

3. McKusick, V.A. Mendelian Inheritance in Man (Johns Hopkins University Press, Baltimore, 1994).

4. Ryan, S.G. et al. Nature Genet. 17 92-95 (1997).

5. Page, D.C., Harper, M.E., Love, J. \& Botstein, D. Nature 311, 119-123 (1984).

6. Sargent, C.A. et al. Genomics 32, 200-209 (1996).

7. Mumm, S., Molini, B., Terreil, J., Srivastava, A. \& Schlessinger, D. Genome Res. in press (1997).

8. Disteche, C. Trends Genet. 11, 17-22 (1995).

9. D'Esposito, M.D. et al. Nature Genet. 13, 227-229 (1996). 